\title{
Looking beyond the thrombus: essentials of pulmonary artery imaging on CT
}

\author{
Mohammed M. Khadir • Apeksha Chaturvedi • \\ Mike S. Nguyen • John C. Wandtke • Susan Hobbs • \\ Abhishek Chaturvedi
}

Received: 6 January 2014 /Revised: 15 May 2014 / Accepted: 28 May 2014 / Published online: 8 July 2014

(C) The Author(s) 2014. This article is published with open access at Springerlink.com

\begin{abstract}
Background Pulmonary arteries are not just affected by thrombus. Congenital and acquired conditions can also involve the pulmonary arteries. An awareness of these conditions is important for the radiologist interpreting chest computed tomography (CT).

Methods The anatomy of the pulmonary arteries was reviewed. CT and magnetic resonance (MR) acquisition protocols for imaging the pulmonary arteries were discussed. The imaging appearances of congenital and acquired anomalies involving the pulmonary arteries, using $\mathrm{CT}$ and other modalities, were presented.

Results Imaging features of congenital anomalies presented include pulmonary agenesis, partial pulmonary artery agenesis, patent ductus arteriosus, pulmonary artery sling, congenital pulmonary artery stenosis and coronary to pulmonary artery fistula. Acquired pulmonary artery anomalies discussed include arteritis, infected aneurysm and sarcoma. Pulmonary artery filling defects besides thromboembolism are also discussed, including foreign body emboli. Imaging features of bronchogenic carcinoma and mediastinal fibrosis demonstrating compression of the pulmonary arteries are presented, followed by a brief discussion of post repair appearance of the pulmonary arteries for congenital heart disease.

Conclusions Congenital and acquired pulmonary artery anomalies have a characteristic appearance on a variety of imaging modalities. An acquaintance with the imaging features of these anomalies is needed to avoid misinterpretation and reach the correct diagnosis.
\end{abstract}

M. M. Khadir $(\triangle) \cdot$ A. Chaturvedi $\cdot$ M. S. Nguyen $\cdot$ J. C. Wandtke

S. Hobbs $\cdot$ A. Chaturvedi

Cardiothoracic Imaging Section, Department of Imaging Sciences,

University of Rochester Medical Center, 601 Elmwood Ave,

Rochester, NY 14642, USA

e-mail: mohammed_khadir@urmc.rochester.edu
Teaching Points

- Discuss a variety of congenital and acquired anomalies of the pulmonary arteries.

- Discuss the imaging appearance of the presented congenital or acquired pulmonary artery anomalies.

- Describe CT and MR acquisition protocols for imaging the pulmonary arteries.

- Review the anatomy of the pulmonary arteries.

Keywords Pulmonary artery · Congenital anomalies . Acquired anomalies $\cdot$ Embryology $\cdot$ Pulmonary embolus

\section{Introduction}

Often, the frontal chest radiograph provides the first clue to the presence of an abnormal pulmonary artery (Fig. 1a). If the pulmonary artery is enlarged, it presents with an enlarged contour of the vessel below the aortopulmonary window. Transverse diameter of the normal right interlobar artery from its lateral aspect to the intermediate bronchus is $15 \mathrm{~mm}$ in women and $16 \mathrm{~mm}$ in men. Computed tomography (CT) with intravenous contrast (Fig. 1b) provides more detail of the lumen, vessel wall and adjacent mediastinal structures. Greater anatomical detail is obtained with magnetic resonance (MR) imaging, allowing for improved evaluation of the vessel wall and quantification of flow (Fig. 1c). It also allows for pulmonary artery maximal and minimal cross-sectional area measurement to be made perpendicular to the axis of blood flow, useful in identifying distensibility (Fig. 1d) [1]. Positron emission tomography (PET)-CT is useful to evaluate for malignancy and arteritis. More invasive methods of imaging the pulmonary artery include intravascular ultrasound and catheter angiography.

In this article, we will briefly review the embryology and anatomy of the pulmonary arteries, followed by a discussion of the CT appearance of the common congenital anomalies 

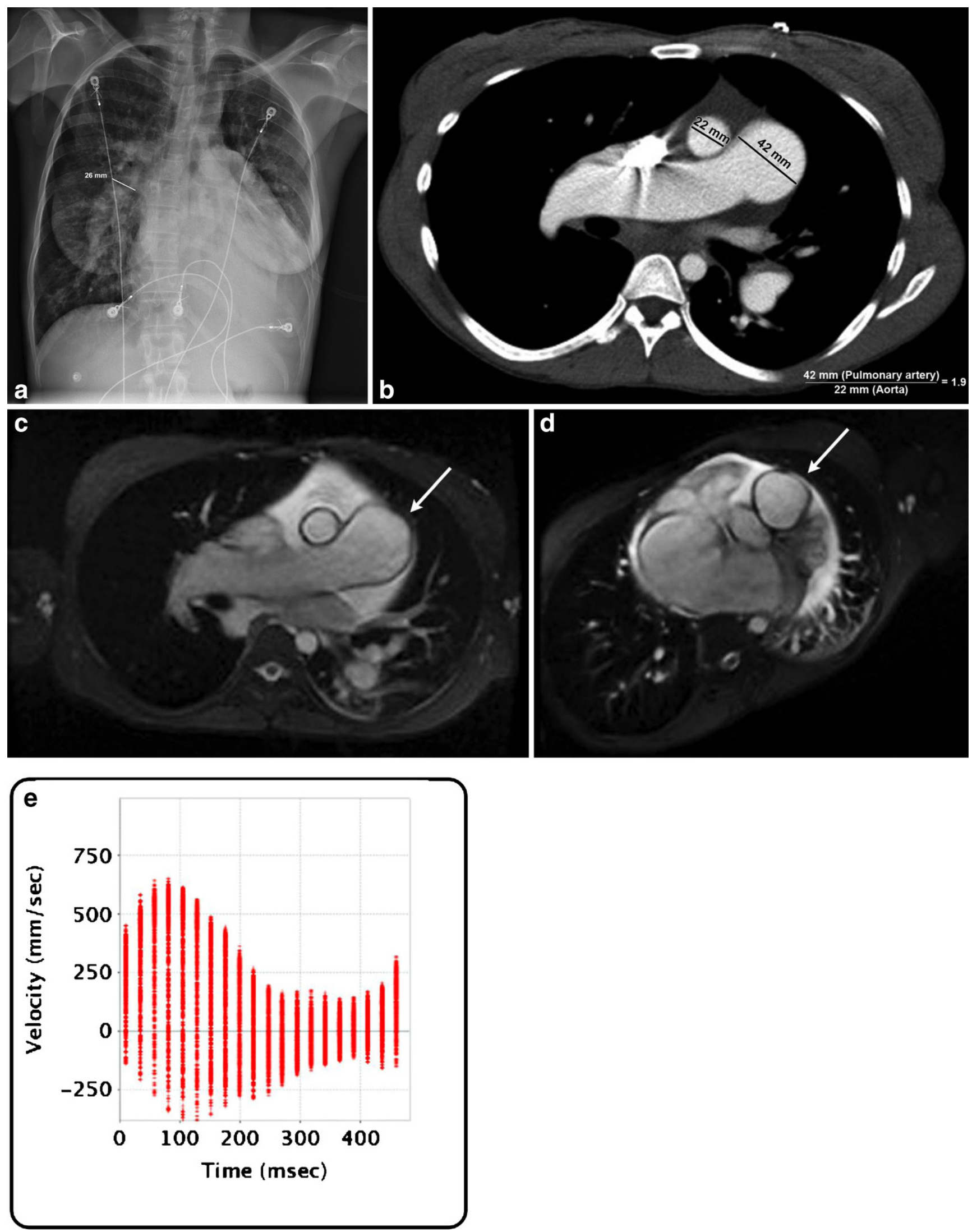
4 Fig. 1 a Frontal radiograph of a patient with sinus venosus atrial septal defect demonstrates a dilated pulmonary artery, pulmonary oedema, left pleural effusion and cardiomegaly. The right lower lobe pulmonary artery measures $26 \mathrm{~mm}$. b Contrast-enhanced axial CT image demonstrates an enlarged main pulmonary artery in the same patient. The pulmonary artery to aorta ratio is 1.9 . $\mathrm{c}$ Axial steady state free precession images in the same patient demonstrate enlarged pulmonary artery (arrow). d Pulmonary artery distensibility $(1.8 \%)$ can be calculated using cine MR, by measuring the diastolic minimal $\left(10.9 \mathrm{~cm}^{2}\right)$ and systolic maximal $\left(11.1 \mathrm{~cm}^{2}\right)$ cross-sectional area obtained perpendicular to direction of blood flow. e Average velocity obtained with phase contrast MR in the main pulmonary artery is $11.6 \mathrm{~cm} / \mathrm{s}$

and acquired conditions affecting the pulmonary arteries. For ease of discussion, the acquired entities will be categorised as those affecting the vessel wall, intraluminal abnormalities and extraluminal abnormalities. In addition, a brief discussion of imaging appearance in patients with repaired congenital heart diseases affecting the pulmonary arteries is also included.

\section{Embryology}

During the 4th-5th week of embryogenesis, the aortic sac gives rise to six paired arteries called the aortic arches, which will eventually develop into the mature aortic arch and other major vessels (Fig. 2). The arches originate from the aortic sac and terminate in the right and left dorsal aorta. The right sixth aortic arch persists as the proximal right pulmonary and the distal main pulmonary artery. The primitive truncus arteriosus forms the proximal main pulmonary artery. The left pulmonary artery and the distal right pulmonary artery develop from arteries arising from the adjacent lung buds and surrounding mesoderm [2].

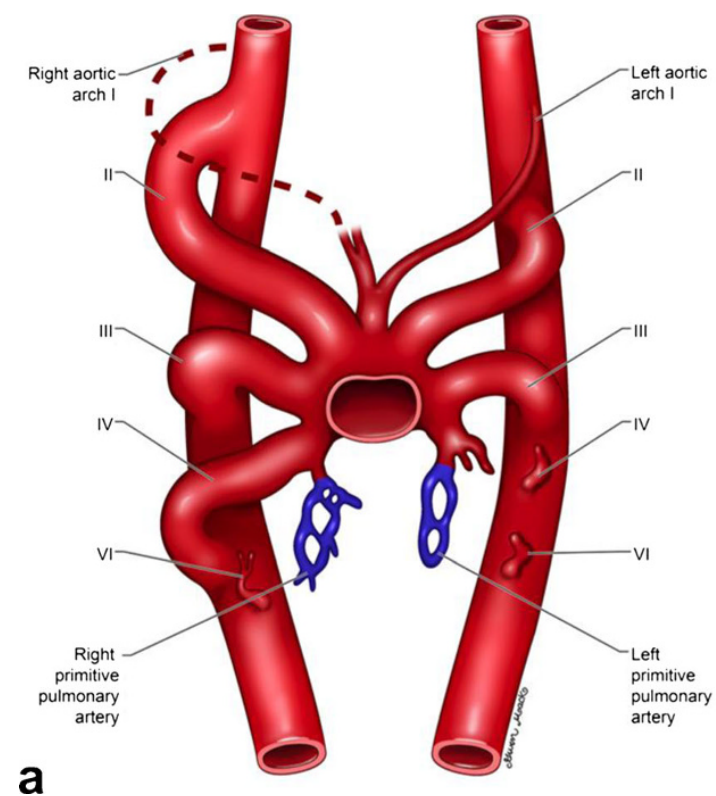

Fig. 2 a Illustrations depicting the developing six paired aortic arches with the left and right dorsal aorta during early embryogenesis. b Further development leads to formation of right and left pulmonary arteries from

\section{Anatomy}

Two arterial circulations supply the lungs [3]. The bronchial circulation draws $1 \%$ of systemic cardiac output and normally only supplies nutrients to the lungs. The primary circulation is the pulmonary arteries, which convey venous blood to the lungs from the heart. A pulmonary artery branch accompanies the bronchial tree and ends in capillary network within the alveolar wall [4]. The normal main pulmonary artery (MPA) divides into the right and left branches before it exits the pericardium. The left pulmonary artery (LPA) travels over the left mainstem bronchus before dividing into its two branches at the root of the left lung. The right pulmonary artery (RPA) continues from the MPA before diving into its two branches, the superior and inferior (interlobar) trunk, at the root of the right lung. The superior trunk supplies the right upper lobe with the interlobar trunk supplying the middle and lower lobes. The lobar branches divide into segmental and subsegmental arteries. Right middle lobe medial and lateral segmental arteries may arise as a common trunk from the interlobar artery or as separate branches. The right lower lobe artery first gives off an apical segmental branch and distal to this the right lower lobe artery is called the basal trunk. Lower lobe artery gives off the medial basal and anterior basal followed by the lateral and posterior basal segmental arteries. On the left, there is no truncus anterior, and the segmental branches originate directly from the LPA. For the left upper lobe and lingual arteries, there may be five to seven segmental branches. The superior segmental artery of the lower lobe arises from the left interlobar artery above the origin of lingular branches. Caudal to this, the left interlobar artery becomes basal trunk

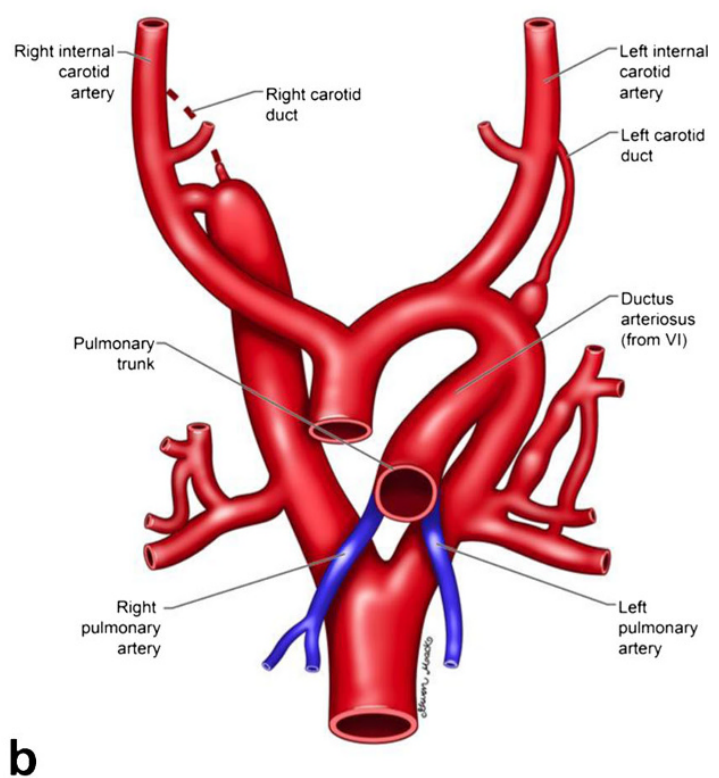

the sixth aortic arches, primitive truncus arteriosus and adjacent arteries. Arrest in this normal development can lead to agenesis, partial agenesis, pulmonary sling, etc. 


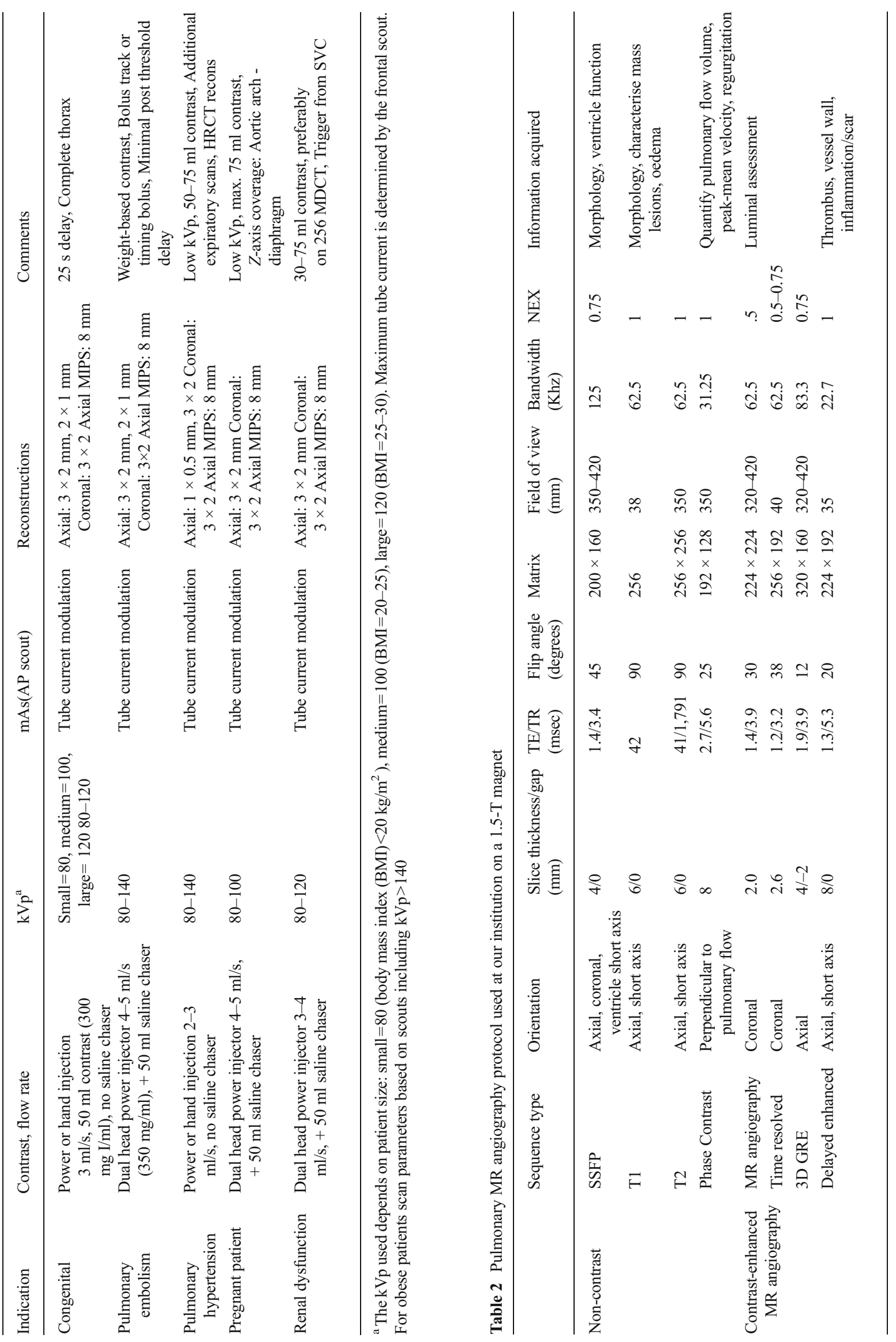


giving rise to lower lobe segmental branches [5]. The basal branches may be duplicated or triplicated [4].

On CT, the main pulmonary artery measures up to $28 \mathrm{~mm}$, some studies have found $29 \mathrm{~mm}$ in men and $27 \mathrm{~mm}$ in women to be the upper limit for normal $[3,6]$. A convenient method to evaluate for pulmonary artery enlargement is to determine whether the ratio of the main pulmonary artery to the ascending aorta (Fig. 1b) is greater than 0.9 [6].

Normal main pulmonary artery pressure ranges from 8 to $20 \mathrm{mmHg}$. In pulmonary hypertension (intraluminal pressure exceeding $25 \mathrm{mmHg}$ at rest or $30 \mathrm{mmHg}$ with exercise), frontal chest radiograph demonstrates a prominent pulmonary artery silhouette with dilated hilar vessels and diminished peripheral vascularity (Fig. 1a). Phase-contrast MR-derived mean average velocity $<11.7 \mathrm{~cm} / \mathrm{s}$ can help in detection of pulmonary hypertension (sensitivity $92.9 \%$ and specificity $82.4 \%$ ) [7]. Pulmonary arterial transit times measured using time-resolved MR angiography can be used as a simple, noninvasive metric for detection of altered haemodynamics in pulmonary arterial hypertension [8]. Cine MR derived pulmonary artery distensibility of $>10 \%$ (systolic pulmonary artery area - diastolic pulmonary artery area $\div$ systolic pulmonary artery area $\times 100$ ) [1] is useful to evaluate pulmonary hypertensive patients who would respond to vasodilator therapy. In patients with Fontan circulation, pulmonary perfusion ratios are more accurately evaluated with phase contrast MR compared with lung perfusion scintigraphy [9].

\section{Acquisition protocols}

Pulmonary CT angiography protocols have been evolving over the years for evaluating pulmonary embolus [10]. Adequate contrast opacification is critical for diagnostic quality, which depends upon patient weight, cardiac output, scan duration and contrast delivery protocol $[11,12]$. Arterial enhancement depends on the amount of contrast delivered per unit of time (injection flow rate) and the injection duration, measured in seconds [13]. Suggested minimal luminal attenuation to see all acute and chronic pulmonary venous emboli $(\mathrm{PE})$ is 93 and $211 \mathrm{HU}$ respectively [14]. On a 64-detector CT, a mean pulmonary artery opacification of $250 \mathrm{HU}$ could be achieved with $1.2 \mathrm{ml} / \mathrm{kg}$ of $350 \mathrm{mg} \mathrm{I} / \mathrm{ml}$ injected at $4 \mathrm{ml} / \mathrm{s}$ [11]. Iodine flow rate of $1.6 \mathrm{~g} \mathrm{I} / \mathrm{s}$ has been suggested as optimal to reach the pulmonary artery enhancement of $300 \mathrm{HU}$ [15]. The scan duration depends upon the scanner $(16,64$, dual source, dual source high pitch, 256, 320 slice multidetector [MD] $\mathrm{CT}$ ), which on a high pitch scanner this may be less than 2 seconds [16]. With a faster scanner, contrast volume can also be decreased by using a higher iodine concentration [12].

For CTA, a region of interest can be placed in the main pulmonary artery and a timing bolus or bolus tracking can be utilised to determine the time it takes for intravenously injected contrast to reach the pulmonary arteries [17]. Either of these techniques results in homogenous opacification and diagnostic image quality [18]. Contrast flow rate of at least $3 \mathrm{ml} / \mathrm{s}$ is associated with lower frequency of insufficient contrast enhancement during chest CT [19]. Flow rate of more than $4 \mathrm{ml} / \mathrm{s}$ using an 18-G cannula has been suggested for PE exams [20,21] A lower volume of contrast and iodine dose can be administered using a higher concentration $(350 \mathrm{mg}$ iodine $/ \mathrm{ml}$ vs $300 \mathrm{mg} / \mathrm{ml}$ ) [22]. Wu et al. [23] have described a low contrast dose $(30 \mathrm{ml})$ pulmonary 64-detector CT angiography technique without compromising diagnostic image quality. The duration of contrast administration is calculated as scan duration plus additional few seconds $(6-8 \mathrm{~s})$. This delay accounts for the interval between the scan trigger and the start of acquisition [12].

When evaluating for Fontan circulation, Park et al. [24] found that a 3-min delay time from the time of injection to be optimal for enhancement of the pulmonary arteries, irrespective of the intravenous route used for administration. Bolus tracking
Fig. 3 Contrast-enhanced CT image (a) shows complete agenesis of left lung and left pulmonary artery. The left hemithorax is smaller with mediastinal shift toward the left and the elevation of the left hemidiaphragm. The abdominal contents are seen in the left hemithorax. Contrast-enhanced axial CT image (b) demonstrates partial agenesis of the left pulmonary artery (arrow) with hypoplasia of left lung. There is no mediastinal shift, but the abdominal organs extend into the thorax
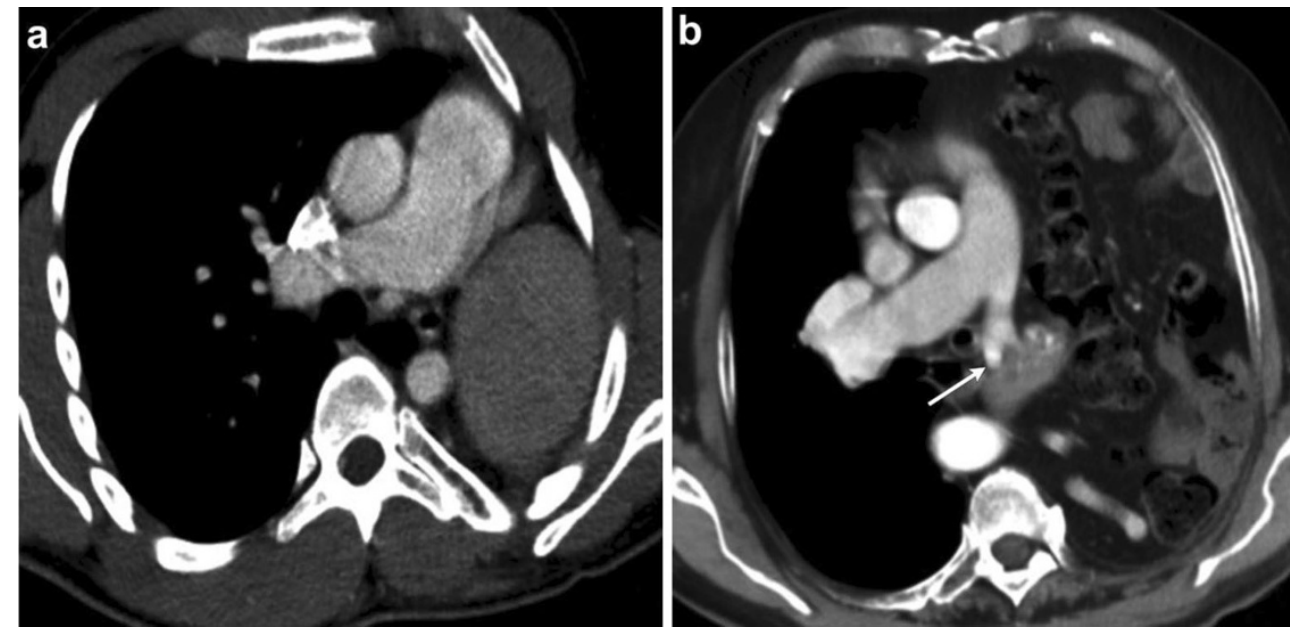

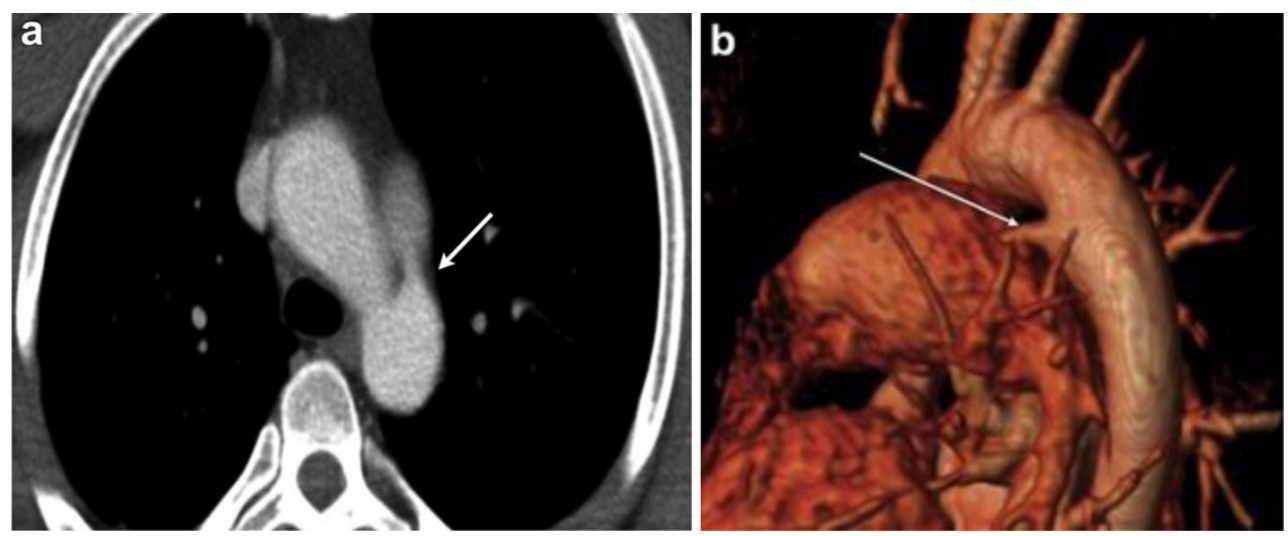

Fig. 4 Contrast-enhanced axial CT image (a) and a volume rendered image (b) in a patient with patent ductus arteriosus $(P D A)$ depicting the persistent communication between the pulmonary artery and descending aorta (arrow). The flow direction in the post-natal period is aorta to

demonstrated a high failure rate in providing homogenous enhancement of the Fontan circulation and of the pulmonary arteries.

For all pulmonary $\mathrm{CT}$ angiography studies, a caudocranial direction of acquisition is recommended as it reduces the chances of having respiratory motion related artefacts [14]. At our institution, in-patients with normal (Stage 1, glomerular filtration rate $[$ GFR $\mathrm{mL} / \mathrm{min} / 1.73 \mathrm{~m} 2]=90+$ ) and mildly reduced renal function (Stage 2, GFR $=60-89$ ) and no contraindication to CT contrast agent, contrast volume is determined from patient height, weight, age, sex, heart rate and scan duration using vendor-specified protocol (MEDRAD) with a timing bolus (test bolus of $20 \mathrm{ml}$ contrast and $50 \mathrm{ml}$ saline at $4 \mathrm{ml} / \mathrm{s}$ to find the time to peak in the main pulmonary artery is used to determine the scan delay, scan delay=time to peak in pulmonary artery $+9 \mathrm{~s}$ ) [25]. The maximum allowed injection flow rate is $6 \mathrm{ml} / \mathrm{s}$. In patients with moderately impaired renal function (stage $3 \mathrm{~A}, \mathrm{GFR}=45-60$ ), bolus tracking with $75 \mathrm{ml}$ of contrast at $4-5 \mathrm{ml} / \mathrm{s}$ is used. In patients with moderately reduced renal function (Stage $3 \mathrm{~B}$, GFR=30-44) $30 \mathrm{ml}$ of contrast with bolus tracking from SVC, preferably on the 256 slice MDCT is used. Any contrast injection is avoided in pulmonary artery as the pulmonary pressures decrease. This can lead to pulmonary hypertension, which on CT will present as enlarged pulmonary trunk as seen on the volume rendered image

patients with GFR less than 29 unless they are on haemodialysis. CT angiography protocol used at our institution is presented in Table 1 .

\section{MR}

MR imaging for the diagnosis of pulmonary artery disease can be performed using high-field MR scanners ( $>1.5 \mathrm{~T})$ [26]. It is indicated when cardiac function and flow needs to be evaluated, such as congenital heart disease, calculating intra/extracardiac shunts, right ventricle strain in PE and pulmonary hypertension. Non-contrast sequences used include a bright blood steady state free precession (SSFP), T2-weighted inversion recovery and T1 GRE (gradient echo). Post-contrast MR angiography is performed with extracellular gadolinium contrast agent injected at $0.1-0.2 \mathrm{mmol} / \mathrm{kg}$. When evaluating for PE, a combination of MR angiography GRE and SSFP images have the highest sensitivity [27]. MR is the imaging modality of choice for evaluating the right ventricle size and function [28]. Contrast-enhanced MR angiography with gadoliniumbased MRI contrast agent, using both high-spatial-resolution and high-temporal-resolution protocols (high-spatial-
Fig. 5 Illustration (a) and contrast-enhanced axial CT image (b) depicting the left pulmonary artery coursing between the trachea and oesophagus to reach the left pulmonary hilum. Patient's symptoms correlate with the degree of upper airway obstruction present from narrowing of the trachea
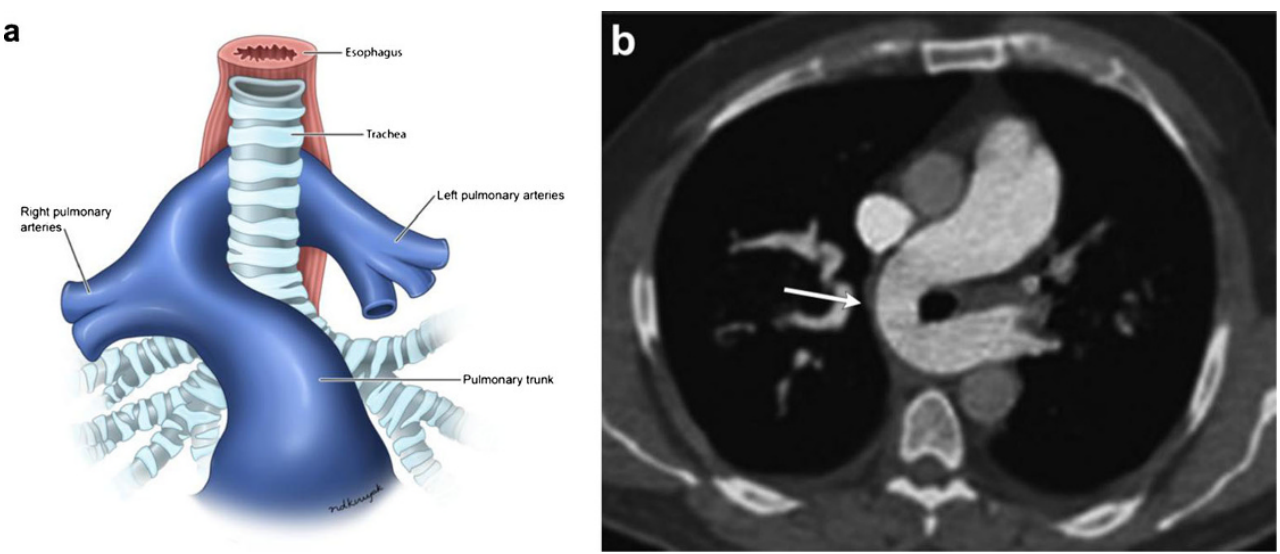
Fig. 6 Contrast-enhanced axial CT image (a) from a patient with tetralogy of Fallot (TOF) and a prosthetic pulmonic valve demonstrates severe stenosis of the left and mild stenosis of the right pulmonary artery (arrows). In addition there is an ascending aortic aneurysm. In a different patient (b) with an unrepaired $T O F$, an aneurysm of the pulmonary trunk (arrow) formed with a chronic thrombus in the right and left pulmonary arteries. Also note the multiple dilated aortopulmonary collaterals. CT angiogram $\mathbf{c}$ performed with $30 \mathrm{ml}$ of contrast in a patient with chronic renal failure and a prosthetic pulmonary valve demonstrates a main pulmonary artery aneurysm (measuring $44 \mathrm{~mm})$
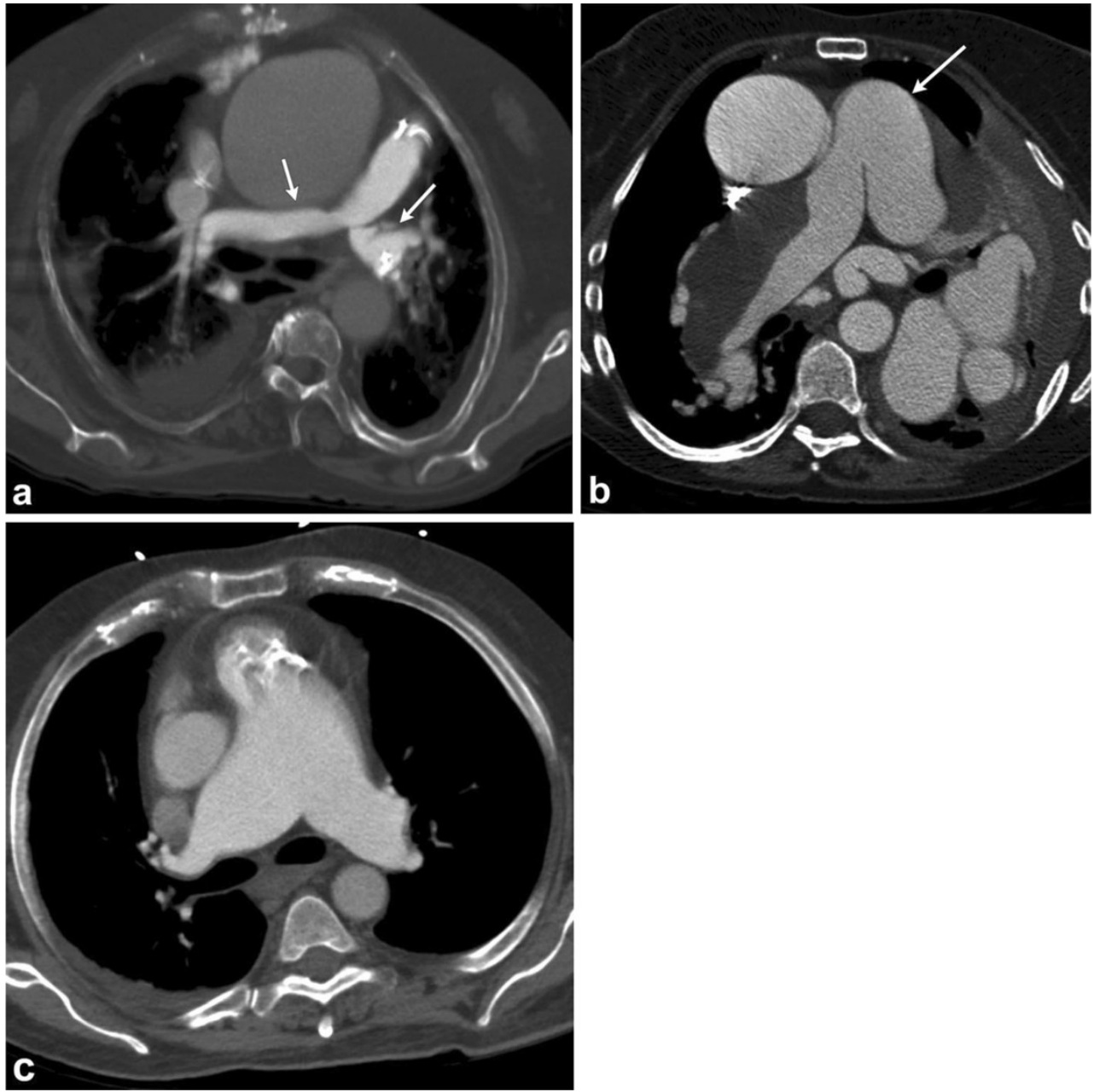

resolution contrast-enhanced MR angiography and timeresolved contrast-enhanced MR angiography), is an excellent non-invasive imaging tool for the evaluation of surgical cavopulmonary connections [29]. Pulmonary MR angiography should be considered as an alternative to CT angiography when iodine contrast injection or radiation is a significant matter [30]. It has been proposed that electrocardiograph (ECG)-gated and respiratory navigator-gated MR angiography at $3 \mathrm{~T}$ using a blood-pool contrast agent at $0.3 \mathrm{mmol} / \mathrm{kg}$ can deliver better image quality and vessel sharpness [31]. Although, gadolinium-based contrast agents are not recommended in patients with a GFR less than 30 or acute renal failure in patients with hepatorenal syndrome unless essential due to risk for nephrogenic systemic sclerosis [32]. Pulmonary MR angiography protocol used at our institution is presented in Table 2.

\section{PET-CT}

F-18 fluorodeoxyglucose (FDG) PET/CT is useful in identifying a pulmonary artery lesion as malignant if the luminal lesion has high FDG uptake [33] and is useful in preoperative

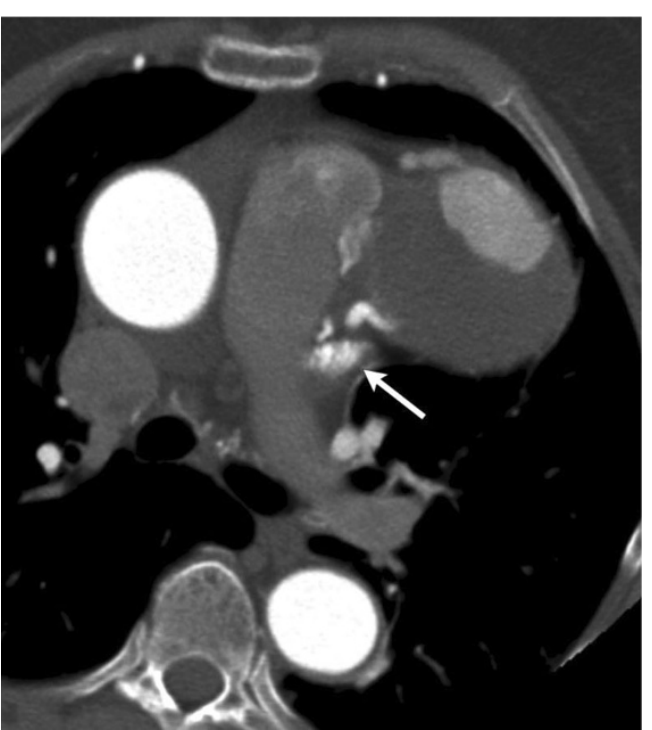

Fig. 7 Contrast-enhanced axial CT image in systemic arterial phase demonstrates contrast blush within the pulmonary trunk emanating from a tubular enhancing structure along the left anterior descending coronary artery. Communication is noted between this and the pulmonary artery, suggesting a coronary to pulmonary artery fistula (arrow) 


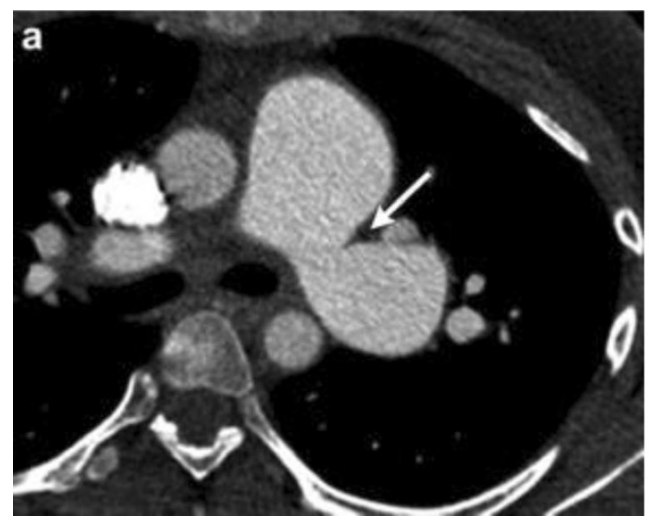

Fig. 8 Contrast-enhanced axial CT image (a) in a 16-year-old patient with progressive dyspnea and absent left upper extremity pulse shows a focus of smooth narrowing and aneurysmal dilatation of the left main pulmonary artery (arrow). Late venous phase axial MR image from a 3D

evaluation [34]. It is also very useful in identifying active vasculitis in patients with pulmonary vasculitis such as Takayasu's arteritis [35] and monitoring response to immunosuppressive treatment [36]. At our institution, a PET-CT for these indications is combined with a contrast-enhanced CT angiography of pulmonary arteries to better depict the vascular anatomy rather than a non-contrast $\mathrm{CT}$ for attenuation correction.

\section{Congenital}

Unilateral pulmonary agenesis presents with unilateral absence of the lung and absence of the ipsilateral pulmonary artery and veins (Fig. 3a). The aetiology is unknown, although genetic factors, viral infections, folate and vitamin A deficiencies have been proposed as possible causes [37]. Newborns with this abnormality typically do not present with respiratory distress, but are likely to have other anomalies associated with the cardiovascular, musculoskeletal or gastrointestinal system. Later in life, patients may have poor lung function with recurrent respiratory infections. CT demonstrates decreased volume in the ipsilateral

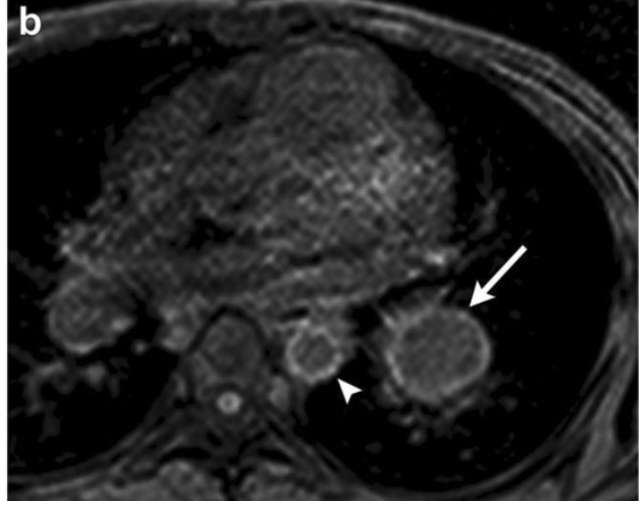

GRE acquisition (b) shows delayed enhancement of an aneurysmal left pulmonary artery branch (arrow). Also note the wall enhancement of descending thoracic aorta (arrowhead) consistent with vasculitis. These findings are suggestive of Takayasu arteritis

hemithorax, complete absence of lung parenchyma, agenesis of pulmonary artery and veins. There is elevation of hemidiaphragm and mediastinal shift to the affected side $[38,39]$.

Partial pulmonary artery agenesis involves an absence of the proximal portion or a rudimentary pulmonary artery. Blood flow to the ipsalateral lung is achieved through collaterals provided from the brachial arteries and transpleural branches of the thoracic arteries. Patients with the anomaly show an increased predisposition to dyspnea, recurrent respiratory infections and pulmonary haemorrhage. Chest radiographs demonstrate ipsilateral volume loss with hyperinflation of the contralateral side. CT illustrates (Fig. 3b) a rudimentary proximal vessel and hypoplastic lung. Transpleural collaterals can be seen as pleural thickening and subpleural parenchymal bands on the CT $[3,40]$.

The primitive left sixth aortic arch gives rise to the ductus arteriosus, which connects the descending thoracic aorta to the left pulmonary artery. Patent ductus arteriosus anomaly arises with persistent postnatal hypoxia, leading to failure of contraction of the ductus with formation of a continuous left to right shunt forms. A small shunt predisposes to endocarditis and a larger shunt causes haemodynamic derangement,
Fig. 9 Contrast-enhanced axial CT (a) in a patient with Bechet's disease demonstrate a focal aneurysm of the right lower lobe pulmonary artery with eccentric mural thrombus (arrow). Volume rendered image (b) better depicts the eccentric saccular aneurysm (arrow). This patient underwent right lower lobectomy for recurrent haemoptysis
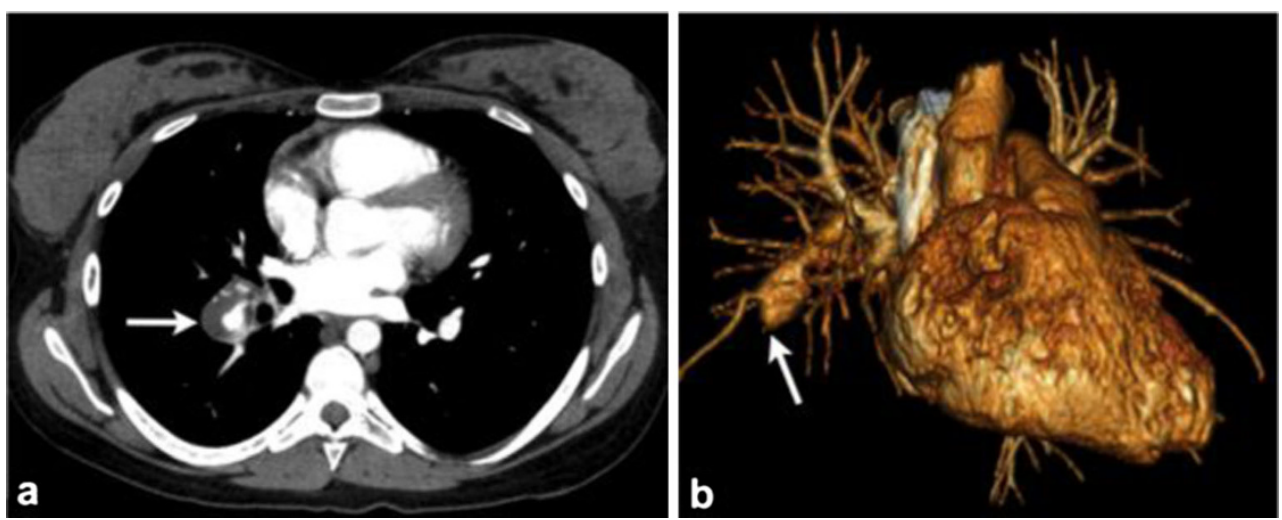


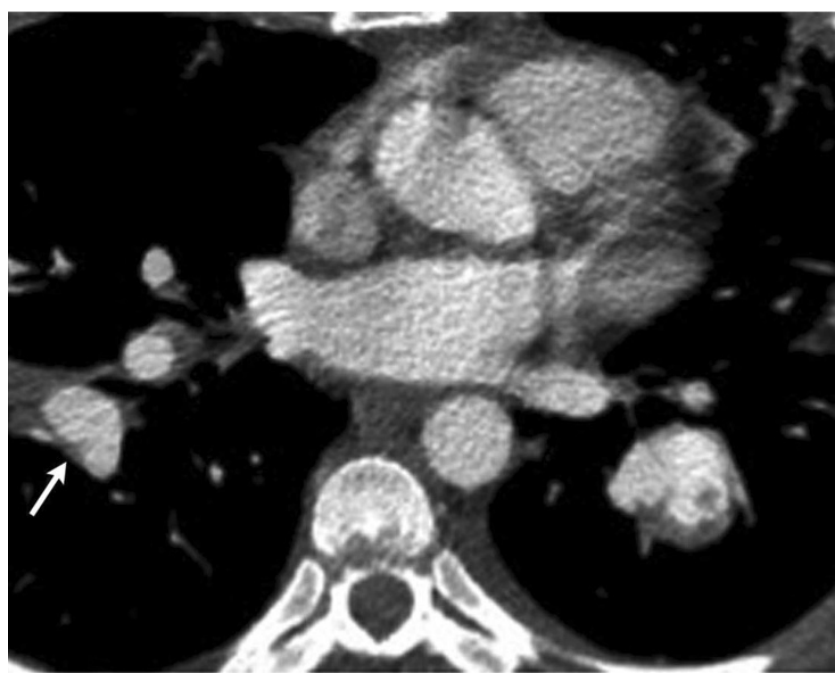

Fig. 10 Contrast-enhanced axial CT image demonstrates aneurysmal formation with irregular thick walls in the segmental branches of right and left lower lobe pulmonary arteries (arrow) in this patient with a known infected aneurysm. These findings were new compared with prior chest CT

eventually leading to Eisenmenger syndrome [41]. Symptomatic patients may present with dyspnea, tachycardia, a widened pulse pressure and a machinery-like continuous murmur. CT demonstrates dilated pulmonary artery, pruning of the peripheral compared with central pulmonary vasculature. Contrast-enhanced CT will identify the patent communication between the descending thoracic aorta and the pulmonary artery. Cardiac MR can be used to quantitate the left to right shunt (Fig. 4) [41, 42].

Pulmonary artery sling presents when the left pulmonary artery arises from the posterior aspect of the right pulmonary artery before coursing between the trachea and oesophagus to reach the left hilum (Fig. 5). The sling around the distal trachea and right mainstem bronchus causes a variable amount of compression of these structures and may lead to stenosis of a long segment of the trachea. The amount of upper airway stenosis correlates to the degree of the patient's symptoms. CT can accurately illustrate the anomaly. In addition, phase contrast MR may be used for quantification of pulmonary blood flow $[3,43]$. Flow measurements are calculated from single slice phase contrast MR obtained perpendicular to MPA, RPA and LPA.

Pulmonary artery stenosis leads to right ventricular outflow tract obstruction and can be secondary to a variety of congenital or acquired aetiologies. In tetralogy of Fallot (TOF), hemodynamic consequences depend largely on the degree of right ventricular outflow tract obstruction, including supravalvular narrowing, which has been reported in up to $50 \%$ of patients [44]. Other congenital aetiologies for pulmonary artery stenosis include Williams syndrome, Alagille syndrome and congenital rubella [45]. Affected regions of the vessel demonstrate fibrous intimal proliferation with loss of elastic fibres, leading to varying degrees of stenosis. Poststenotic segments may be dilated or aneurysmal and often is the first clue on radiographs. A pulmonary artery aneurysm is
Fig. 11 Contrast-enhanced axial CT image (a) demonstrates a large filling defect in the left pulmonary artery (arrow). The lesion remained stable after a course of anticoagulation, which raised the suspicion for a malignancy.

Subsequently obtained FDG-PET (b) demonstrated the central part of this filling defect to be hypermetabolic, consistent with a primary pulmonary artery sarcoma. Gadolinium-enhanced cardiac MR (c) performed $60 \mathrm{~s}$ post contrast for preoperative evaluation demonstrates a lesion in the left pulmonary artery with an non-enhancing central portion, consistent with a bland thrombus, and an enhancing component in the pulmonary arteries and left lower lobe, suggestive of a tumour
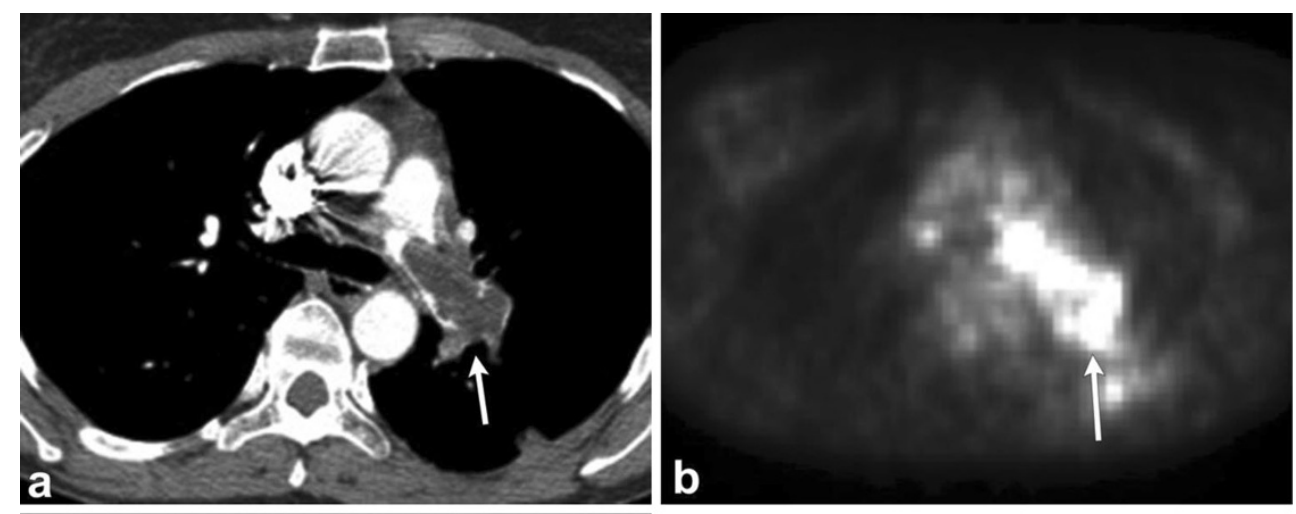

b

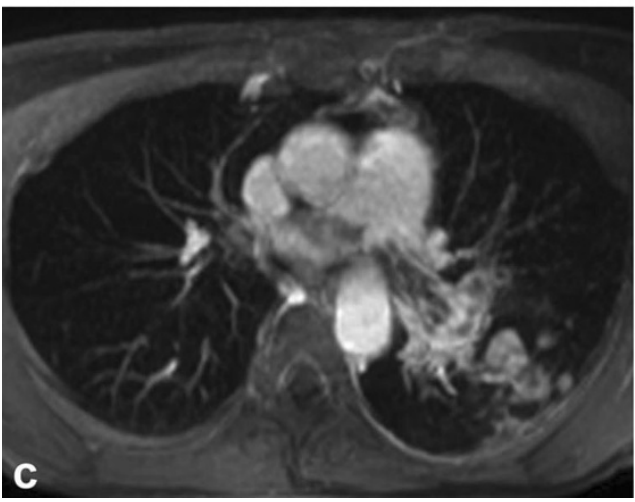


Fig. 12 Different patients with non-thrombotic emboli to the pulmonary arteries: catheter fragment (a), non-target emboli from $N$-butyl-2-cyanoacrylate injection of gastric varices (b), inferior vena cava filter prong (c) and bone cement for vertebropasty (d)
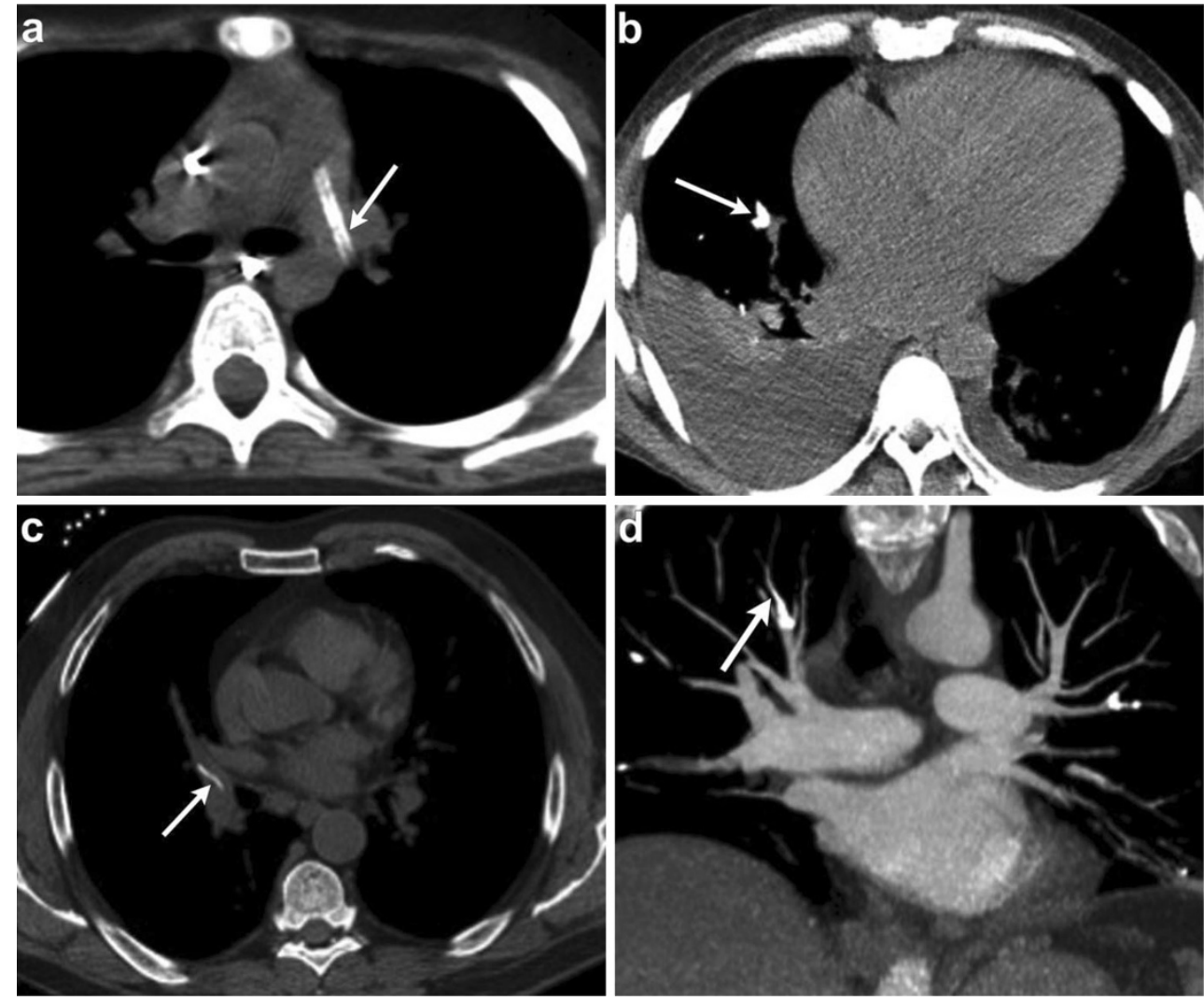

commonly defined as the pulmonary trunk measuring more than $4.5 \mathrm{~cm}$ and the right or left pulmonary artery measuring greater than $3 \mathrm{~cm} \mathrm{[46].} \mathrm{CT} \mathrm{can} \mathrm{demonstrate} \mathrm{stenosis} \mathrm{in} \mathrm{the}$ main and branch pulmonary arteries with dilated post-stenotic segment (Fig. 6) [47].

Coronary to pulmonary artery fistula is an anomaly that accounts for $15-30 \%$ of all coronary artery fistulas [48]. The fistulous communication can either be congenital or acquired, as in the case of trauma, endovascular procedures and cardiac transplantation. In a few patients, a significant shunt can form, leading to congestive heart failure from volume overload or angina. Most reported cases have been incidentally detected during catheter angiography, but more recently $\mathrm{CT}$ angiography has been used to describe the features of the fistula. Both modalities demonstrate a direct communication between the two vessels. If the $\mathrm{CT}$ images are acquired in the systemic arterial phase, the only finding will be a contrast blush within the pulmonary artery (Fig. 7).

\section{Acquired}

Acquired diseases affecting the vessel wall include vasculitis, infected aneurysm and sarcoma. Takayasu arteritis is an idiopathic disorder producing granulomatous inflammation of the arterial wall. It involves the pulmonary artery is $50-80 \%$ of cases. In early disease, the vessel wall may demonstrate enhancement and thickening, and in advanced disease, may demonstrate stenosis or occlusion [3, 49]. CT (Fig. 8) demonstrates wall enhancement, stenosis, ectasia or aneurysm of the affected vessels. Behcet disease is a chronic multisystem small vessel vasculitis that can cause aneurysmal dilatation of

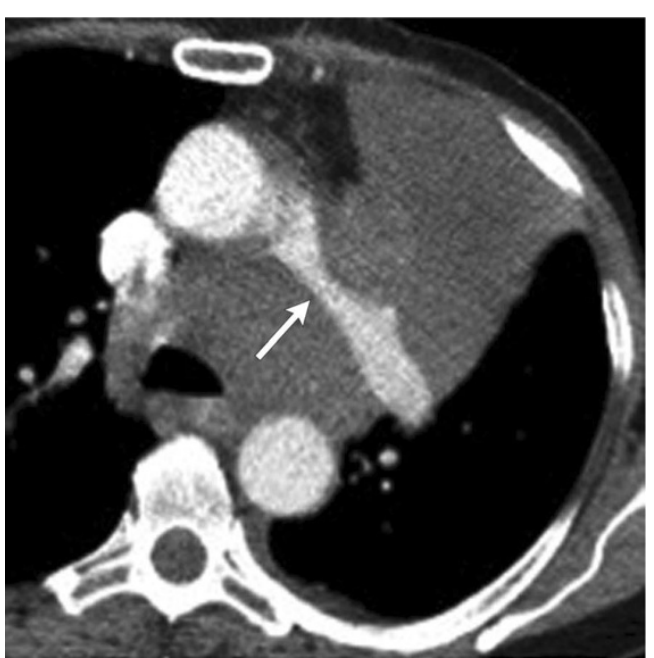

Fig. 13 Contrast-enhanced axial CT image in a patient with left hilar lung cancer demonstrates the left main pulmonary artery being completely encased and narrowed by the left upper lobe mass (arrow), which also extends into the mediastinum 


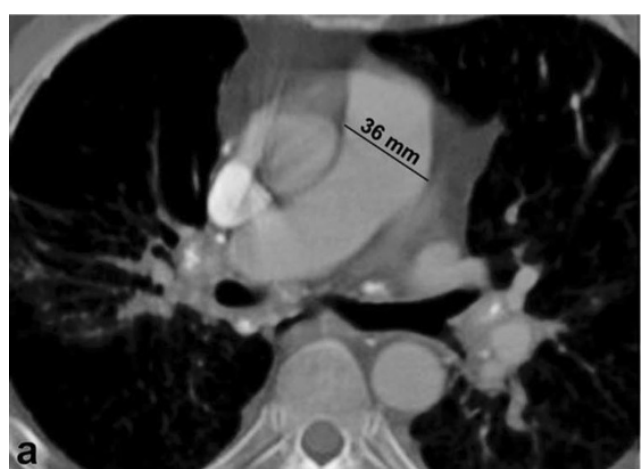

Fig. 14 Axial CT images in a patient with prior histoplasmosis demonstrates an enlarged pulmonary trunk $(36 \mathrm{~mm})$. The proximal right and left pulmonary arteries are normal in calibre but taper and are severely

the pulmonary artery (Fig. 9). MR can be useful in demonstrating wall inflammation in either of these diseases [49].

Infected (mycotic) aneurysm of the pulmonary artery can develop from haematogenous seeding of the infectious agent or continuous involvement from an adjacent source. Staphylococcus, Streptococcus and Salmonella are most often the infectious agents. CT angiogram (Fig. 10) is the modality of

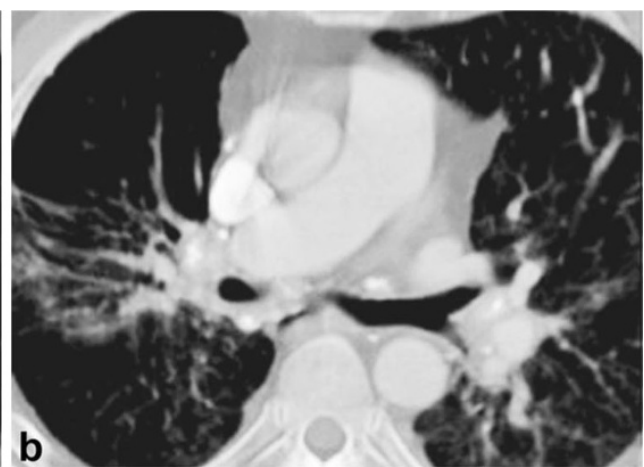

narrowed at the level of hila. In addition, there are calcified mediastinal lymph nodes, calcified pulmonary granulomas and interlobular interstitial thickening. These findings represent fibrosing mediastinitis

choice for evaluation of the infected aneurysm and demonstrates a lobulated vascular mass with an irregular wall arising from the vessel in question. In addition, the soft-tissues surrounding the aneurysm may demonstrate enhancement [50].

Pulmonary artery sarcoma arises from the mesenchymal cells of the intima. On initial evaluation, the entity is often misdiagnosed as a pulmonary embolism because of similar
Fig. 15 Illustration (a) demonstrates stage 1 , the Norwood procedure, for correcting hypoplastic left heart syndrome with the creation of a neoaorta from the pulmonary artery. Post-repair images (b) have a characteristic appearance with a rudimentary proximal ascending aorta and the proximal main pulmonary artery (arrow) reconstituting flow to the distal ascending aorta (arrowhead). During the procedure, the pulmonary trunk is ligated and the pulmonary arterial flow is reestablished from either the subclavian artery or the brachiocephalic trunk. After completion of stage $3(\mathbf{c}, \mathbf{d})$, by attaching the inferior vena cava to the right pulmonary artery, the Fontan procedure, complete systemic venous flow is directed through the right pulmonary artery into the lungs. Note the right pulmonary artery (arrow) shows higher attenuation secondary to the contrast injection from the right arm veins compared with the left pulmonary artery (arrowhead) which has lower attenuation due to blood flow from the inferior vena cava
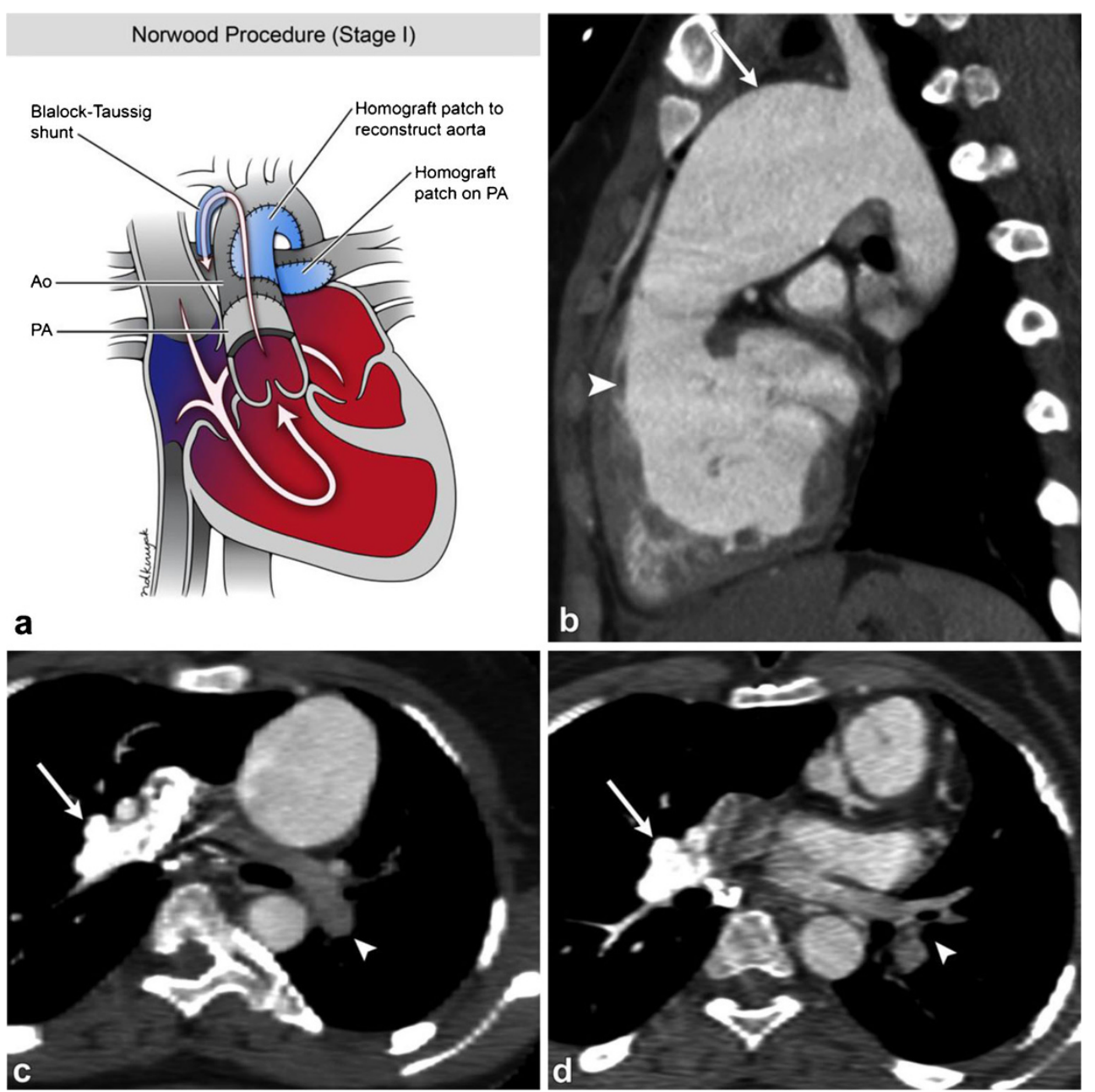
Fig. 16 Contrast enhanced axial CT images in a patient with transposition of great vessels demonstrates the characteristic appearance post-arterial switch. The pulmonary arteries (arrow) are positioned anterior to the aorta with the left and right main branches draping around the aorta. There is a higher incidence of pulmonary artery stenosis in these patients
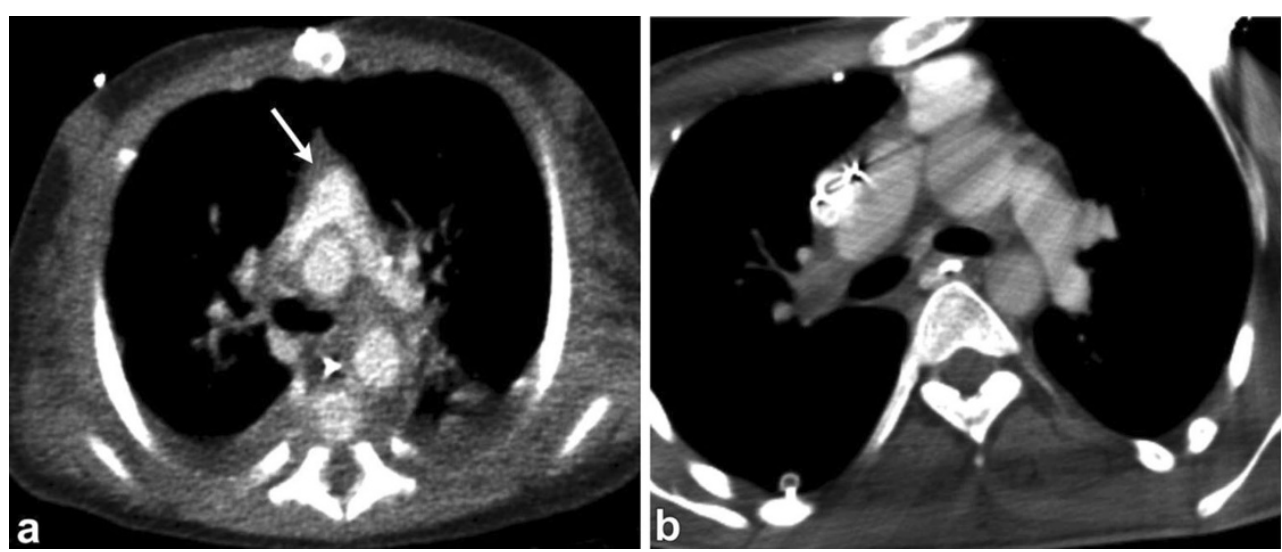

presentation. The two entities can be differentiated using a contrast-enhanced CT by evaluating for a low-attenuation filling defect occupying the entire lumen and leading to expansion of the artery or with extraluminal tumour extension (Fig. 11a) [3]. FDG-PET shows the sarcoma to have higher metabolic activity than blood pool [33] (Fig. 11b).

The majority of intraluminal filling defects of the pulmonary artery are secondary to pulmonary thromboembolism. Several malignancies, including breast and colorectal carcinoma, metastasise to the lungs by the way of the pulmonary arteries. Intraluminal enhancing filling defects of the pulmonary arteries in these patients may represent metastases. In addition, the pulmonary arteries maybe the site for nonthrombotic emboli, such as non-target embolisation of intravascular glue, broken embolised fragments of an IVC filter or vertebroplasty cement (Fig. 12).

The pulmonary artery can also be affected by extrinsic processes. Luminal narrowing of the pulmonary artery may be due to extrinsic compression from bronchogenic carcinoma (Fig. 13), lymphadenopathy or mediastinal fibrosis encasing the vessel [3]. Pulmonary artery dilatation can be seen with pulmonary hypertension, which can be secondary to a pulmonary parenchymal disease. CT is essential in evaluating the lung parenchyma and, in addition, will demonstrate pulmonary artery diameter greater than $28 \mathrm{~mm}$ or a pulmonary artery to ascending aorta transverse diameter ratio greater than $0.9[6,51]$. Granulomatous fibrosing mediastinitis is an infiltrative disorder that results from excessive fibrosis in the mediastinum, usually a sequela of histoplasmosis (Fig. 14). It can result in encasement of the mediastinal viscera with narrowing of the vessels, airway, and other mediastinal structures [52].

Corrective surgical procedures for congenital cardiovascular diseases which affect the pulmonary arteries result in a characteristic appearance. Cavopulmonary shunts or Fontan circulation are used to treat infants with single effective ventricle (tricuspid/pulmonary atresia, hypoplastic left heart/hypoplastic right heart syndrome). The venous return is diverted to the pulmonary arteries bypassing the morphological right ventricle. The Norwood procedure is used to correct hypoplastic left heart syndrome, which is frequently associated with hypoplasia of the ascending aorta. Stage 1 involves creating a neoaorta from the proximal main pulmonary artery, which is connected to the ascending aorta (Figs. 15a and $b$ ). The right subclavian artery or the brachiocephalic trunk is then connected to the right pulmonary artery to provide blood flow to the lungs. Stage 2 of the procedure creates a Glenn shunt, a superior cavopulmonary shunt from an end-to-end anastomosis between the superior vena cava and right pulmonary artery, thus directing systemic venous flow directly to the lungs. Stage 3 creates a total cavopulmonary connection by attaching the inferior vena cava to the right pulmonary artery, referred to as a Fontan procedure [53, 54] (Figs. 15c and d). Contrast timing during pulmonary CT angiography is critical in such patients to when evaluating for a suspected stenosis or PE.

An arterial switch is performed for treating transposition of great arteries. It results in a characteristic appearance of the main pulmonary artery situated anterior to the ascending aorta with the right and left pulmonary arteries draped around the aorta. This repair can be associated with narrowing of the pulmonary arteries (Fig. 16).

\section{Conclusion}

Congenital and acquired pulmonary artery anomalies have a characteristic appearance on a variety of imaging modalities. Even though imaging findings on CT were mainly discussed, the interpreting radiologist needs to be familiar with findings of these entities on a spectrum of imaging modalities to avoid misinterpretation and reach the correct diagnosis.

Conflict of interest No potential conflicts of interest to disclose. 


\section{Appendix}

Table 3 Maximum contrast volume for CT angiography using power injector is based on patient weight (iodine concentration of $350 \mathrm{mg} / \mathrm{ml})$

\begin{tabular}{ll}
\hline Weight $(\mathrm{kg})$ & Contrast volume $(\mathrm{ml})$ \\
\hline 40 & 69 \\
45 & 77 \\
50 & 86 \\
55 & 95 \\
60 & 103 \\
65 & 112 \\
70 & 120 \\
75 & 129 \\
80 & 137 \\
85 & 143 \\
90 & 143 \\
\hline
\end{tabular}

Open Access This article is distributed under the terms of the Creative Commons Attribution License which permits any use, distribution, and reproduction in any medium, provided the original author(s) and the source are credited.

\section{References}

1. Jardim C et al (2007) Pulmonary artery distensibility in pulmonary arterial hypertension: an MRI pilot study. Eur Respir J 29(3):476-481

2. Abdulla R, Blew GA, Holterman MJ (2004) Cardiovascular embryology. Pediatr Cardiol 25(3):191-200

3. Castaner E et al (2006) Congenital and acquired pulmonary artery anomalies in the adult: radiologic overview. Radiographics 26(2): 349-371

4. Grey H (2000) In: WH Lewis (ed) Anatomy of the human body, 20th edn.. Lea \& Febiger, New York

5. Kadir S. Pulmonary arterial and venous anatomy. Atlas of normal and variant angiographic anatomy. Philadelphia: W.B. Saunders Company

6. Truong QA et al (2012) Reference values for normal pulmonary artery dimensions by noncontrast cardiac computed tomography: the Framingham heart study. Circ Cardiovasc Imaging 5(1):147-154

7. Sanz J et al (2007) Pulmonary arterial hypertension: noninvasive detection with phase-contrast MR imaging. Radiology 243(1):70-79

8. Jeong HJ et al (2011) Time-resolved magnetic resonance angiography: evaluation of intrapulmonary circulation parameters in pulmonary arterial hypertension. J Magn Reson Imaging 33(1):225-231

9. Fratz S et al (2002) More accurate quantification of pulmonary blood flow by magnetic resonance imaging than by lung perfusion scintigraphy in patients with fontan circulation. Circulation 106(12):1510-1513

10. Sadigh G, Kelly AM, Cronin P (2011) Challenges, controversies, and hot topics in pulmonary embolism imaging. AJR Am J Roentgenol 196(3):497-515

11. Bae KT et al (2007) Effect of patient weight and scanning duration on contrast enhancement during pulmonary multidetector CT angiography. Radiology 242(2):582-589

12. Ramos-Duran LR et al (2010) Current contrast media delivery strategies for cardiac and pulmonary multidetector-row computed tomography angiography. J Thorac Imaging 25(4):270-277
13. Fleischmann D (2006) Contrast medium applications for multislice CT. In: Bruening R, Küttner A, Flohr T (eds) Protocols for multislice CT, 2nd edn. Springer, Berlin

14. Wittram C (2007) How I do it: CT pulmonary angiography. AJR Am J Roentgenol 188(5):1255-1261

15. Schoellnast $\mathrm{H}$ et al (2006) MDCT angiography of the pulmonary arteries: influence of body weight, body mass index, and scan length on arterial enhancement at different iodine flow rates. AJR Am J Roentgenol 187(4):1074-1078

16. Lell M et al (2009) High-pitch electrocardiogram-triggered computed tomography of the chest: initial results. Invest Radiol 44(11):728-733

17. Bae KT (2005) Test-bolus versus bolus-tracking techniques for CT angiographic timing. Radiology 236(1):369-370, author reply 370

18. Kerl JM et al (2012) Intravenous contrast material administration at high-pitch dual-source $\mathrm{CT}$ pulmonary angiography: test bolus versus bolus-tracking technique. Eur J Radiol 81(10):2887-2891

19. Ozawa Y, Hara M, Shibamoto Y (2011) The frequency of insufficient contrast enhancement of the pulmonary artery in routine contrastenhanced chest CT and its improvement with an increased injection rate: a prospective study. J Thorac Imaging 26(1):42-47

20. Bae KT, Tran HQ, Heiken JP (2000) Multiphasic injection method for uniform prolonged vascular enhancement at CT angiography: pharmacokinetic analysis and experimental porcine model. Radiology 216(3):872-880

21. Browne AM et al (2014) Evaluation of imaging quality of pulmonary 64-MDCT angiography in pregnancy and puerperium. AJR Am J Roentgenol 202(1):60-64

22. Goble EW, Abdulkarim JA (2014) CT pulmonary angiography using a reduced volume of high-concentration iodinated contrast medium and multiphasic injection to achieve dose reduction. Clin Radiol 69(1):36-40

23. Wu CC et al (2012) Pulmonary 64-MDCT angiography with $30 \mathrm{~mL}$ of IV contrast material: vascular enhancement and image quality. AJR Am J Roentgenol 199(6):1247-1251

24. Park EA et al (2010) Optimal scan timing and intravenous route for contrast-enhanced computed tomography in patients after fontan operation. J Comput Assist Tomogr 34(1):75-81

25. CR D (2013) A clinical evaluation of an automated software program (P3T Cardiac) for patient specific contrast injection during chest CTA to exclude pulmonary embolism

26. Junqueira FP et al (2012) Pulmonary arterial hypertension: an imaging review comparing MR pulmonary angiography and perfusion with multidetector $\mathrm{CT}$ angiography. $\mathrm{Br} \mathrm{J}$ Radiol 85(1019):1446-1456

27. Kalb B et al (2012) MR imaging of pulmonary embolism: diagnostic accuracy of contrast-enhanced 3D MR pulmonary angiography, contrast-enhanced low-flip angle 3D GRE, and nonenhanced freeinduction FISP sequences. Radiology 263(1):271-278

28. Pena E et al (2012) Pulmonary hypertension: how the radiologist can help. Radiographics 32(1):9-32

29. Wagner M et al (2012) Contrast-enhanced MR angiography of cavopulmonary connections in adult patients with congenital heart disease. AJR Am J Roentgenol 199(5):W565-W574

30. Pleszewski B et al (2006) Gadolinium-enhanced pulmonary magnetic resonance angiography in the diagnosis of acute pulmonary embolism: a prospective study on 48 patients. Clin Imaging 30(3):166172

31. Dabir D et al (2012) High-resolution motion compensated MRA in patients with congenital heart disease using extracellular contrast agent at 3 tesla. J Cardiovasc Magn Reson 14:75

32. Schlaudecker JD, Bernheisel CR (2009) Gadolinium-associated nephrogenic systemic fibrosis. Am Fam Physician 80(7):711714

33. Chong S et al (2007) Pulmonary artery sarcoma mimicking pulmonary thromboembolism: integrated FDG PET/CT. AJR Am J Roentgenol 188(6):1691-1693 
34. Tueller C et al (2010) FDG-PET in diagnostic work-up of pulmonary artery sarcomas. Eur Respir J 35(2):444-446

35. Addimanda $\mathrm{O}$ et al (2013) Pulmonary artery involvement in Takayasu arteritis. PET/CT versus CT angiography. Clin Exp Rheumatol 31(1 Suppl 75):S3-S4

36. Karapolat I et al (2013) Comparison of F18-FDG PET/CT findings with current clinical disease status in patients with Takayasu's arteritis. Clin Exp Rheumatol 31(1 Suppl 75):S15-S21

37. Currarino G, Williams B (1985) Causes of congenital unilateral pulmonary hypoplasia: a study of 33 cases. Pediatr Radiol 15(1): $15-24$

38. Greenough A, Ahmed T, Broughton S (2006) Unilateral pulmonary agenesis. J Perinat Med 34(1):80-81

39. Espinosa L, Agarwal P (2008) Adult presentation of right lung agenesis and left pulmonary artery sling. Acta Radiol 49(1):41-44

40. Keiffer SA et al (1965) Proximal interruption of a pulmonary artery. Am J Roentgenol 95(3):592-597

41. Wang ZJ et al. (2003) Cardiovascular shunts: MR imaging evaluation. Radiographics 23 Spec No:S181-S194

42. Berko NS, Haramati LB (2012) Simple cardiac shunts in adults. Semin Roentgenol 47(3):277-288

43. Siripornpitak S et al (1997) Pulmonary artery sling: anatomical and functional evaluation by MRI. J Comput Assist Tomogr 21(5):766768

44. Mirowitz SA et al (1989) Tetralogy of fallot: MR findings. Radiology 171(1):207-212

45. Warnes CA et al (2008) ACC/AHA 2008 guidelines for the management of adults with congenital heart disease: executive summary: a report of the american college of cardiology/american heart association task force on practice guidelines (writing committee to develop guidelines for the management of adults with congenital heart disease). Circulation 118(23):2395-2451

46. Restrepo CS, Carswell AP (2012) Aneurysms and pseudoaneurysms of the pulmonary vasculature. Semin Ultrasound CT MR 33(6):552566

47. Warnes CA et al (2008) ACC/AHA 2008 guidelines for the management of adults with congenital heart disease: a report of the american college of cardiology/american heart association task force on practice guidelines (writing committee to develop guidelines on the management of adults with congenital heart disease). Circulation 118(23):e714-e833

48. Tomasian A et al (2008) Coronary artery to pulmonary artery fistulae with multiple aneurysms: radiological features on dual-source 64-slice CT angiography. Br J Radiol 81(969): e218-e220

49. Castaner E et al (2010) When to suspect pulmonary vasculitis: radiologic and clinical clues. Radiographics 30(1):33-53

50. Lee WK et al (2008) Infected (mycotic) aneurysms: spectrum of imaging appearances and management. Radiographics 28(7):18531868

51. Frazier AA, Burke AP (2012) The imaging of pulmonary hypertension. Semin Ultrasound CT MR 33(6):535-551

52. McNeeley MF et al (2012) Imaging of granulomatous fibrosing mediastinitis. AJR Am J Roentgenol 199(2):319-327

53. Gaca AM et al (2008) Repair of congenital heart disease: a primerpart 1. Radiology 247(3):617-631

54. Bardo DM et al (2001) Hypoplastic left heart syndrome. Radiographics 21(3):705-717 